\title{
Adipose tissue-derived stem cell expansion on collagen/ chitosan scaffolds in bioreactor
}

\author{
Yanxia Zhu ${ }^{1}$, Tianqing Liu ${ }^{1}$, Kedong Song ${ }^{1}$, Xiubo Fan ${ }^{1}$, Lili Jiang ${ }^{1}$, Xuehu Ma ${ }^{1}$, Zhanfeng Cui ${ }^{2}$ \\ ${ }^{1}$ Dalian R\&D Center for Stem Cell and Tissue Engineering, Dalian University of Technology, Dalian 116024, China; ${ }^{2}$ Oxford \\ Centre for Tissue Engineering and Bioprocessing, Department of Engineering Science, Oxford University, Oxford OXI 3PJ, UK
}

Recent reports indicate that adipose tissue is a novel source of multipotent stem cells, which can be used in cell therapy and tissue engineering. However, the static amplification of adipose tissue-derived stem cells(ADSCs) is a time-consuming procedure and prone to contamination, and it's hard to meet the need of clinical applications. To obtain the stem cells in a great number of quantity and with stemness well reserved, we plated ADSCs in collagen/ chitosan scaffolds and compared the proliferation of ADSCs in three dimensional (3D) static environment and 3D dynamic environment. hADSCs were isolated and cultivated in T-flask, in collagen/chitosan scaffolds statically or in the scaffolds placed in spinner flasks for 21 days. During the culture period, the growth dynamic parameters and viability of ADSCs were examined by CCK-8 kit every other day; metabolic rates of glucose and lactic acid were analyzed every day. After 21 days, the cells were evaluated with scanning electron microscope (SEM) for histological examination of the aggregates. The surface markers (CD13, CD29, CD34, CD44, CD45, CD105, CD166 and HLADR), the specific transcription factors (Oct-4, Sox-2, Rex-1) and the multi-differentiation potential (adipogenic, osteogenic and chondrogenic) were also assayed to identify the stemness of proliferated cells. The results showed that in spinner flask, the cells in scaffolds could be expanded by more than ten times, and they presented better morphology and vitality and stronger differentiation ability than cells cultivated in scaffolds statically. And the expansion of the both cases for cells in scaffolds was better than that in T-flask. All cells maintained stem cell characteristics after proliferation. From the results, we found that dynamic spinner flask cultivation of hADSC/ scaffold constructs resulted in increased proliferation, differentiation and distribution of cells in scaffolds. Therefore, spinner flask cultivation is an easy-to-use inexpensive system for expanding hADSCs in 3D scaffolds.

Keywords: adipose tissue, mesenchymal stem cells, scaffolds, bioreactor, expansion, collagen/chitosan Cell Research (2008) 18:s164. doi: 10.1038/cr.2008.254; published online 4 August 2008

Correspondence: Tianqing Liu

E-mail: liutq@dlut.edu.cn 